is naturaleza 
Para la publicación de este número se ha contado con la ayuda

financiera de las siguientes instituciones:

Departamento de Filosofía y Lógica y Filosofía de la Ciencia de la Universidad de Sevilla

Facultad de Filosofía de la Universidad de Sevilla 


\section{NATURALEZA Y LIBERTAD}

Revista de estudios interdisciplinares

Número 4

Málaga, 2014

Esta revista es accesible on-line en el siguiente portal:

http://grupo.us.es/naturalezayl 


\section{Naturaleza y Libertad}

Revista de estudios interdisciplinares

Número 4

ISSN: 2254-96682014

Directores: Juan Arana, Universidad de Sevilla; Juan José Padial, Universidad de Málaga; Francisco Rodríguez Valls, Universidad de Sevilla.

Secretaria: Avelina Cecilia Lafuente, Universidad de Sevilla.

Consejo de Redacción: Jesús Fernández Muñoz, Universidad de Sevilla; José Luis González Quirós, Universidad Juan Carlos I, Madrid; Francisco Soler, Universität Dortmund / Universidad de Sevilla; Pedro Jesús Teruel, Universidad de Valencia; Héctor Velázquez, Universidad Panamericana, México.

Adjunto a la redacción: Miguel Palomo, Universidad de Sevilla

Consejo Editorial: Mariano Álvarez, Real Academia de Ciencia Morales y Políticas; Allan Franklin, University of Colorado; Michael Heller, Universidad Pontificia de Cracovia; Manfred Stöcker, Universität Bremen; William Stoeger, University of Arizona.

Consejo Asesor: Rafael Andrés Alemañ Berenguer. Universidad de Alicante; Juan Ramón Álvarez, Universidad de León; Luciano Espinosa, Universidad de Salamanca; Miguel Espinoza, Université de Strasbourg; Juan A. García González, Universidad de Málaga; José Manuel Giménez Amaya, Universidad de Navarra; Karim Gherab Martín, Urbana University, Illinois; Martín López Corredoira, Instituto de Astrofisica de Canarias; Alfredo Marcos, Universidad de Valladolid; Marta Mendonça, Universidade Nova de Lisboa; Javier Monserrat, Universidad Autónoma de Madrid; Leopoldo Prieto, Colegio Mayor San Pablo, Madrid; Ana Rioja, Universidad Complutense, Madrid. Madrid; José Luis González Recio, Universidad Complutense, Madrid; Javier Serrano, TEC Monterrey (México); Hugo Viciana, Université Paris I; Claudia Vanney, Universidad Austral, Buenos Aires; José Domingo Vilaplana, Huelva.

\section{Redacción y Secretaria:}

Naturaleza y Libertad. Revista de estudios interdisciplinares. Departamento de Filosofia y Lógica. Calle Camilo José Cela s.n. E-41018 Sevilla.

Depósito Legal: MA2112-2012

욜 954.55.77.57 Fax: 954.55.16.78. E-mail: jarana@us.es

(C) Naturaleza y Libertad. Revista de Filosofía, 2014 


\section{ÍNDICE}

\section{ESTUDIOS}

Manuel Alfonseca (Universidad Autónoma de Madrid), Diseño inteligente, evolución al azar, o evolución providencial....... ..11

Paul Gilbert, S J (U. Gregoriana, Roma), Deseo de conocer ..............................................27

Javier Hernández-Pacheco (U. de Sevilla), Evolución, erotismo y origen de las especies. De vuelta desde Darwin a Platón y Aristóteles.

Miguel Ángel Herrero (U. Politécnica de Madrid), La formación de los conceptos científicos. De Grosseteste a Galileo.

Ma Teresa Pelacho López (Zaragoza), Otra autonomía de la ciencia. Reflexiones a la luz del pensamiento de Serguei Kara-murzá.......

Juan Fernando Sellés (U. de Navarra), Estudio de las tesis centrales de Millán-Puelles sobre la libertad....... 189

Francisco Soler Gil (U. de Sevilla), El naturalismo y la tentación de las extrapolaciones omnicomprensivas.

Héctor Velázquez Fernández (U. Panamericana, México), En torno a la naturalización de la conciencia sugerida por Daniel Dennett

\section{SECCIÓN BIBLIOGRÁFICA}

Maria Camila Gallego: Falsos saberes.

Juan Arana: Bernardino Telesio, La naturaleza según sus propios principios. 
Naturaleza y Libertad. Revista de estudios interdisciplinares. Número 4, 2014. ISSN: 2254-9668

\title{
LA FORMACIÓN DE LOS CONCEPTOS CIENTÍFICOS. DE GROSSETESTE A GALILEO
}

\author{
Miguel Ángel Herrero \\ Universidad Politécnica de Madrid
}

Resumen Está ampliamente admitido que la finalidad de la ciencia experimental es la descripción de los fenómenos naturales mediante el lenguaje matemático. Para lo cual, es preciso definir conceptos sobre entidades observables que puedan ser medidas experimentalmente. La historia del pensamiento científico pone de manifiesto que la formación de este tipo de conceptos exigió abandonar ciertas categorías filosóficas que restringían la capacidad de la ciencia para el conocimiento empírico de la naturaleza. A la superación de ese enfoque metafísico enraizado en el pensamiento aristotélico, contribuyeron algunos destacados investigadores vinculados al Merton College de Oxford, a la Universidad de París y muy especialmente Galileo. En este trabajo hemos examinado el desarrollo histórico del pensamiento científico mediante algunos ejemplos, tales como la formalización geométrica de la propagación de la luz de Grosseteste, el concepto de ímpetus según la física medieval y en particular la aplicación del método de Galileo al estudio del movimiento acelerado.

Palabras clave: Conceptos científicos, formalización matemática, historia de la ciencia, método de Galileo.

Abstract: It is widely accepted that the aim of experimental science is to describe natural phenomena by mathematical language. This task implies the definition of concepts about observable entities capable of being measured by experimental means. The history of sci- 
Naturaleza y Libertad. Revista de estudios interdisciplinares. Número 4, 2014. ISSN: 2254-9668

entific thought shows that the construction of these concepts required denying some philosophical categories that restricted the role of science in the empirical knowledge of nature. Outstanding scholars from Merton College in Oxford, along with others from the University of Paris, and mainly Galileo, contributed to overcome the metaphysical schemes rooted in the Aristotelian doctrine. In this paper, the historical development of scientific thought has been analyzed with references to a few examples, such as the geometrical modeling of light transmission by Grosseteste, the concept of impetus according to medieval physics, and above all, the use of the Galilean method as applied to the study of accelerated movement.

Key words: Scientific concepts, mathematical modeling, history of science, Galilean method.

Recibido: 21/09/2013. Aprobado: 08/11/2013

\section{INTRODUCCIÓN}

El estilo dialógico de muchas de las obras de Galileo no es sólo un hábil artificio literario para captar el interés del lector. Los largos debates que mantienen Salviati, Sagredo y Simplicio son también un trasunto de las encendidas polémicas que el científico italiano mantuvo con algunos académicos y filósofos de su época. En algunos casos, tales discusiones derivaron en enemistades personales. Pero también estimularon su fértil imaginación, pues, —como él mismo reconoció— "la ignorancia de sus adversarios" había sido su mejor maestra ${ }^{1}$.

1 S. Drake, Galileo: Pioneer Scientist, Toronto, University of Toronto Press, 1990, pp. 169 y ss. 
Las ideas surgidas de esas confrontaciones dialécticas libres del fragor de la disputa adquirieron la mesura y el rigor del texto científico que es posible apreciar en la elegante prosa del Dialogo y los Discorsi ${ }^{3}$. Así nació el Discorso intorno alle cose che stanno in su l'acqua o que in quella si muovono ${ }^{4}$. Este ensayo científico fue impreso en 1612 y concebido en el verano de 1611, en el transcurso de varios debates que Galileo mantuvo con un grupo de profesores de la Universidad de Pisa encabezados por Ludovico delle Colombe. A esta publicación siguió Istoria e dimistrazioni intorno alle macchie Solari, sobre las manchas solares. En ella refutaba la tesis del matemático alemán Christoph Scheiner que, siguiendo la doctrina aristotélica sobre la perfecta esfericidad de los cuerpos supralunares, atribuía tales manchas a la existencia de minúsculos planetas que giraban alrededor del Sol.

Pero de todas las controversias que encendieron el ánimo polémico de Galileo, la que tuvo mayor repercusión fue la provocada por la publicación impresa en Perugia, en el año 1619, titulada Libra Astronómica ac Philosopbica, cuyo autor era el matemático Orazio Grassi, que escribía bajo el seudónimo de Sarsi. En ella, se abordaba el análisis de la constitución mate-

2 Galileo, Dialogo sopra $i$ due massimi sistema del mondo. Le Opere di Galileo Galilei, 20 vols. Edizione Nazionale, a cargo de A. Favaro. Firenze, Barbèra, 1890-1909 (1968), Vol. 7.

3 Galileo, Discorsi e dimostrazioni matematiche intorno a due nuove scienze. Le Opere, vol. 8, pp. 203-204.

4 Galileo, Le Opere, Vol. 4, pp. 63-140. 
rial de los cometas, utilizando argumentos incompatibles con el sistema copernicano, defendido desde 1610 por Galileo.

La respuesta del científico italiano no fue inmediata, pues no se hizo pública hasta 1623 y, a juzgar por el tono utilizado, no invitaba a un pacífico diálogo sobre la cuestión. Galileo tituló su escrito Il Saggiatore y su interés rebasa el terreno estrictamente científico para encuadrarse en el marco de la teoría sobre el método científico. Entre otros temas, analiza la tarea de los sentidos en la observación y enuncia el principio que debe guiar la actividad científica en la indagación de la naturaleza.

La filosofía está escrita en este libro grandísimo que continuamente tenemos abierto ante los ojos (quiero decir el universo), pero no se puede entender si antes no se aprende a entender la lengua y a conocer las letras en que está escrito. Está escrito en lengua matemática, y las letras son triángulos, círculos y otras figuras geométricas, y sin estos medios resulta imposible que los hombres entiendan nada: sin ellos, no habría más que un vano dar vueltas por un oscuro laberinto ${ }^{5}$.

Según esta opinión, se asigna a la ciencia la tarea de construir un lenguaje capaz de descifrar los enigmas encerrados en los fenómenos naturales. Se admite que el libro de la naturaleza ya está escrito, y el cometido de la ciencia debe ser construir el lenguaje científico que lo haga comprensible. Pero la 1984 , p. 61. 
actividad del científico no es comparable a la libre creación literaria. Y, en contra de la opinión de Sarsi, tampoco se somete a ninguna autoridad académica.

Me parece, por lo demás, que Sarsi tiene la firme convicción de que para filosofar es necesario apoyarse en la opinión de cualquier célebre autor, de manera que si nuestra mente no se esposara con el razonamiento de otra, debería quedar estéril e infecunda; tal vez piensa que la filosofía es como las novelas producto de la fantasía de un hombre, como por ejemplo la Odisea o el Orlando furioso, donde lo menos importante es que aquello que en ellas se narra sea cierto. Señor Sarsi, las cosas no son asít.

En consecuencia, a juicio del físico toscano, la ciencia empírica se nutre de los datos extraídos de la observación experimental y no tiene su origen en la libre imaginación de la mente humana. Es una actividad de la razón que no admite más coacciones que las impuestas por los hechos naturales y sólo ante ellos debe responder.

La genuina aportación de Galileo al método científico no consistió tanto en trazar el camino preciso que debía seguir la investigación, como en fijar cuál debía ser el punto de partida del recorrido. Se ha señalado que el misterio de los misterios reside en la capacidad del ser humano para aprender e

6 Galileo, El Ensayador. Traducción, prólogo y notas: José Manuel Revuelta, Madrid, Sarpe, 1984, p. 60-61. 
interpretar el lenguaje en que está escrito el libro de la naturaleza ${ }^{7}$, tratándose sobre todo de un lenguaje cuyos signos deben ser construidos a partir de las observaciones experimentales.

Ese complejo proceso de construcción que lleva a cabo la ciencia, implica trasladar las impresiones sensoriales captadas en la observación al mundo del lenguaje simbólico. Esta trasposición de las sensaciones a los signos matemáticos debe hacerse pasando a través de las definiciones conceptuales, ya que las impresiones sensoriales se sitúan en un ámbito natural distinto del mundo conceptual de la matemática. En consecuencia, los conceptos creados por la razón forman el puente que une ambos mundos; el de los datos sensibles, por un lado, y el de las representaciones simbólicas, por otro.

Mediante las palabras utilizando el lenguaje común cuya significación conceptual nos es familiar, estamos capacitados para describir un paisaje o cualquier otro suceso que observamos. Pero la descripción de los fenómenos científicos no puede hacerse utilizando las palabras del lenguaje natural. En éste, los significados de los términos se refieren a la captación sensible externa y no sirven para dar una explicación científica. Por ejemplo, si queremos describir el movimiento de un cuerpo que cae sometido a la atrac-

7 "El misterio de los misterios no es que la naturaleza esté escrita en un lenguaje u otro, sino que el hombre sea capaz de aprenderlo e interpretarlo" (J. Arana, “¿Es la naturaleza un libro escrito en caracteres matemáticos?”, en: Anuario Filosófico, 2000 (33), p. 65. Citando a J. D. Barrow, ¿̇Por qué es el mundo matemático?, Barcelona, Grijalbo, 1997, pp. 98-99). 
Naturaleza y Libertad. Revista de estudios interdisciplinares. Número 4, 2014. ISSN: 2254-9668

ción gravitatoria, deberemos construir un lenguaje, comenzando por definir conceptos adecuados capaces de captar la razón interna de ese fenómeno.

Es evidente que esta compleja operación de construcción conceptual no es completamente libre, pues se origina en la observación experimental y, a partir de ella, abstrae aquellas propiedades que son relevantes para la investigación en cuestión. Así por ejemplo, surgen las definiciones de magnitudes mecánicas, tales como la fuerza, la velocidad o el momento mecánico que tienen una precisa significación en su propio ámbito. Todas ellas proceden de la observación sensible, pero, en ningún caso puede decirse que se identifiquen con impresiones sensibles. Su naturaleza es diferente, pues tales magnitudes son construcciones mentales y, aunque de origen sensible, una vez definidas, las magnitudes físicas mensurables podrán ser equiparadas a las entidades matemáticas.

En consecuencia, de acuerdo con la visión del método iniciado por Galileo, la descripción matemática del mundo empírico exige la construcción de objetos de pensamiento que sean cuantificables y que estén vinculados a las percepciones. Desde luego, el paso de las observaciones a la enunciación de leyes no es inmediato, ni existen tampoco métodos definidos para lograrlo. Es un proceso creativo, imaginativo, (intuitivo en el sentido al que se refiere 
Einstein $^{8}$ ), sobre todo, en las primeras etapas que recorre la investigación hasta llegar a la construcción de la teoría.

A lo largo de los siglos hubo un lento progreso técnico que facilitó la observación experimental y con ello impulsó el estudio matemático de la naturaleza. Los investigadores comprendieron la importancia de centrarse en los aspectos mensurables. En definitiva, se fue abriendo paso lentamente un razonamiento fundamentado en propiedades cuantitativas, mientras que las nociones de tipo cualitativo y las categorías metafísicas perdieron terreno.

Esta larga evolución conceptual hubo de superar obstáculos de distinto tipo. Algunos de ellos tenían su origen en planteamientos teóricos de raíz aristotélica. No pocas veces, las mismas cuestiones debatidas como propias de la filosofía de la naturaleza habían nacido en un ámbito estrictamente filosófico o incluso teológico, como se comprueba en los primeros estudios acerca de la propagación de la luz o sobre la naturaleza del calor. Así lo atestiguan

8 La intuición considerada como actitud mental según la cual la inteligencia se esfuerza en profundizar en el sentido de la experiencia física, familiarizándose con ella. Haciendo referencia a los principios de la investigación, Einstein define la intuición en los términos siguientes: "no hay otro camino lógico que conduzca [a las leyes básicas más generales] sino la sola intuición, que se fundamenta en la penetración profunda de la experiencia" [Cursiva original]. Citado por M. Paty en: Einstein Philosophe, Paris, Presses Universitaires de France, 1993, p. 458. 
Naturaleza y Libertad. Revista de estudios interdisciplinares. Número 4, 2014. ISSN: 2254-9668

los resultados de la investigación histórica llevada a cabo por autores como Alexander Koyré ${ }^{9}$, Pierre Duhem ${ }^{10}$ y, posteriormente, por Sylla ${ }^{11}$ y Wallace ${ }^{12}$.

En las páginas siguientes nos referiremos brevemente a esa evolución del pensamiento científico anterior a Galileo, mostrando mediante ejemplos algunos de los intentos realizados por diversos autores para definir nociones científicas mensurables. No pretendemos trazar una línea, siquiera aproximada, para seguir la evolución histórica de los conceptos científicos, suponiendo que tal tarea fuera posible. Pues, durante siglos el estudio de la naturaleza desprovisto de un método científico fue como "un vano dar vueltas por un oscuro laberinto"13. Nuestro objetivo es intentar comprender cómo fue posible avanzar hacia la salida del laberinto.

Por tanto, tan sólo presentamos algunos ejemplos que permiten comprobar la deriva hacia los conceptos cuantitativos que a lo largo de los siglos se produce en la investigación de la naturaleza. Ante todo, intentaremos preci-

9 A. Koyré, Estudios de historia del pensamiento científico, México, Siglo veintiuno editores, 1990.

10 P. Duhem, Le système du monde. Histoire des doctrines cosmologiques de Platon a Copernic, Paris, Hermann, 1959, vol. 10.

11 E. D. Sylla, The Oxford Calculators and the Mathematics of Motion 1320-1350. Physics and Measurement by Latitudes, New York \& London, Garland Publising Inc., 1991.

12 W. A. Wallace, "Mechanics from Bradwardine to Galileo", en: Journal of the History of Ideas, 1971 (32), no 1 pp. 15-28.

13 Galileo, El Ensayador. Traducción, prólogo y notas: José Manuel Revuelta, Madrid, Sarpe, 1984, p. 61. 
sar cuál es la aportación de Galileo en el modo de estudiar los fenómenos naturales. Su contribución representa un punto de inflexión en esa investigación y sólo a partir de él es posible referirse con propiedad al método científico, entendido como el camino que conduce a la formalización matemática de la naturaleza.

Comenzamos por referirnos a uno de los obstáculos que el pensamiento pre-científico debió superar hasta alcanzar un lenguaje dotado de categorías propiamente científicas. Esta primera dificultad tuvo su origen en una rígida concepción aristotélica de la ciencia conocida en la bibliografía con el término griego de metabasis ${ }^{14}$. Posteriormente, analizamos el intento de presentar mediante conceptos geométricos el fenómeno de propagación de la luz llevado a cabo por Grosseteste, en el siglo XIII. A continuación señalamos algunas de las aportaciones medievales en torno al movimiento local originadas en las universidades de Oxford y París. Finalmente, subrayamos los rasgos característicos que, a nuestro parecer, definen el método de Galileo.

14 S. J. Livesey, "The Oxford Calculators, Quantification of Qualities and Aristotle's Prohibition of Metabasis", en: Vivarium, 1986 (24), p. 51. 
Naturaleza y Libertad. Revista de estudios interdisciplinares. Número 4, 2014. ISSN: 2254-9668

\section{METABASIS Y CIENCIAS SUBALTERNAS}

Según la noción aristotélica, cada ciencia se asienta en determinados principios establecidos, a partir de los cuales siguiendo un razonamiento lógico alcanza su objeto propio. Así por ejemplo, la matemática tiene como objeto de conocimiento el estudio de la cantidad, abstraída de las propiedades sensibles de los cuerpos materiales. El objeto de la física es el estudio de los sucesos naturales sin prescindir de las cualidades sensibles, tales como el movimiento, el calor o la luz.

La validez de una ciencia reside en su unidad interna, esto es, en la coherencia lógica de sus razonamientos desarrollados en consonancia con sus principios. Y puesto que cada ciencia debe guardar fidelidad a sus propios axiomas, no le está permitido desviarse de su objeto de conocimiento. En consecuencia, no será legítima aquella demostración que en su camino se desvíe, pasando de un género de ciencia a otro diferente (metabasis allos genos), pues, eso supondría abandonar el dominio que le ha sido asignado de acuerdo con su objeto propio.

Las consecuencias en la investigación de la naturaleza, derivadas de esta prescripción aristotélica sobre el papel de la ciencia, han sido analizadas por varios autores, como S. J. Livesey, partiendo del siguiente párrafo contenido en el capítulo 7 del libro I de los Analíticos Posteriores. 
Naturaleza y Libertad. Revista de estudios interdisciplinares. Número 4, 2014. ISSN: 2254-9668

No es posible demostrar pasando de un género <a otro>, v. g.: <demostrar > lo geométrico por la aritmética ${ }^{15}$.

A pesar de esa fuerte restricción, en este mismo capítulo, el texto aristotélico admite excepciones, aceptando la existencia de ciencias pertenecientes a géneros "no enteramente diferentes, como son la aritmética y la geometría". De este modo, y manifiestamente en contra de su aserto anterior, Aristóteles concluye su argumentación admitiendo la existencia de ciencias subalternadas. Esto es, determinadas ciencias, tales como las ciencias demostrativas, que estarán exentas de la prohibición mencionada.

Por eso no es posible demostrar mediante la geometría que la ciencia de los contrarios es una sola, pero tampoco que dos cubos son un cubo, ni [es posible mostrar] lo propio de una ciencia mediante otra, a no ser que todas las cosas en cuestión estén subordinadas, las unas a las otras, v. g.: las cuestiones ópticas respecto a la geometría, y las armónicas respecto a la aritmética ${ }^{16}$ [Cursiva añadida].

15 Aristóteles, Tratados de lógica II (Órganon). Sobre la interpretación. Analíticos primeros. Analiticos segundos. Introducción, traducción y notas: Miguel Candel Sanmartin, Madrid, Gredos, 1988, An. Post. Libro I cap. 7; 75 a 38 - 40.

16 Aristóteles, Tratados de lógica II (Órganon). Sobre la interpretación. Analiticos primeros. Analiticos segundos. Introducción, traducción y notas: Miguel Candel Sanmartin, Madrid, Gredos, 1988, An. Post. Libro I cap. 7; 75 b 10-15). 
En los primeros siglos de la investigación científica, las ciencias no tenían asignado un campo de estudio bien definido y no era fácil discernir si un determinado saber cumplía con los requisitos necesarios para ser una ciencia subalternada. En algunos casos, dependiendo del fenómeno natural estudiado, esa tarea podía simplificarse. Así, la geometría, por sus características, se presta al estudio de la propagación rectilínea de la luz. Mientras que las proporciones numéricas de la aritmética son aplicables al estudio de las vibraciones sonoras. Por tanto, la óptica y la acústica pueden tomarse como ciencias subordinadas de la geometría y de la aritmética, respectivamente.

En consecuencia, a pesar de que la categórica separación conceptual decretada por la teoría aristotélica no favorecía el estudio matemático de los fenómenos naturales, la noción de ciencias subalternadas ${ }^{17}$ permitió ampliar el horizonte de investigación y la descripción de la naturaleza en términos cuantitativos, pero, a condición de utilizar la ciencia demostrativa adecuada al caso concreto estudiado.

Algunas publicaciones de autores medievales revelan los debates suscitados en torno a las posibles ciencias demostrativas. Así, por ejemplo, Walter

17 "Otro modo de diferir la ciencia propter quid y la ciencia quia se refiere al tipo de conocimiento que se adquiere mediante cada una ellas. Sobre la misma cosa, una produce conocimiento propter quid y la otra, un conocimiento quia. La relación entre ellas es tal que, una es la ciencia subordinante (subalternans) y la otra, subordinada (subalternata)" (A.C. Crombie, Robert Grosseteste and theorigins of experimental science 1100-1700, Oxford, ClarendonPress, 1962, p. 91). 
Burley, fellow del Merton College hacia 1305 que desarrolló parte de su carrera científica en París y Aviñón, era partidario de una transición descendente de géneros científicos. Es decir, consideraba legítimo pasar de una ciencia demostrativa situada en un nivel superior a otra situada en un nivel inferior. Esto era admisible en dos casos, en primer lugar, cuando el objeto estudiado por una ciencia coincide en parte con el de la otra. Por ejemplo, la ciencia que estudia los triángulos también incluye en su objeto de investigación el estudio de los triángulos isósceles ${ }^{18}$. En segundo lugar, si coinciden en parte los objetos de estudio de dos ciencias, entonces, era legítimo transferir las demostraciones de una a la otra. Por ejemplo, estaría permitido utilizar las propiedades geométricas de la línea para aplicarlas a la “línea visual”1920.

18 S. J. Livesey, "The Oxford Calculators, Quantification of Qualities and Aristotle's Prohibition of Metabasis", en: Vivarium, 1986 (24), p.56.

19 S. J. Livesey, "The Oxford Calculators, Quantification of Qualities and Aristotle's Prohibition of Metabasis", en: Vivarium, 1986 (24), p.56.

20 "La ciencia que estudia los haces de líneas radiantes y las figuras cae bajo el dominio de la geometría, que esa la que concierne el estudio de las líneas y las figuras; la ciencia que se ocupa de la construcción de máquinas, como la arquitectura y otras artes mecánicas, cae bajo la ciencia que estudia la figura de los cuerpos; la ciencia de la armonía cae bajo la aritmética; y la ciencia que utilizan los marinos para navegar por la posición de los astros está subordinada a la astronomía". (A. C. Crombie, Robert Grosseteste and the origins of experimental science 11001700. Oxford at the Clarendon Press, p. 91). 
Estos dos sencillos ejemplos ponen de manifiesto las dificultades que la ciencia incipiente debió superar hasta llegar a la descripción matemática de los fenómenos naturales. La superación de muchas de esas dificultades exigía abandonar la senda trazada por la entonces vigente teoría aristotélica sobre la ciencia. Al mismo tiempo, también era necesario avanzar en la observación experimental, lo cual implicaba progresar en la construcción de dispositivos de laboratorio que permitiesen realizar medidas experimentales.

Desde otra perspectiva, William Ockham admitía la utilización de una ciencia demostrativa que, al servicio de otra ciencia, fuese capaz de llegar a conclusiones válidas. Así, en el prólogo del tratado que titula Ordinatio ${ }^{21}$ concede a la matemática la función de una estructura gramatical auxiliar puesta al servicio de las ciencias empíricas.

Pero en el camino iniciado hacia la formalización matemática surgió un nuevo obstáculo que la investigación medieval hubo de afrontar. En esta ocasión, la dificultad tenía que ver con la utilización de las proporciones numéricas. El problema fue estudiado por Thomas Bradwardine en su Tractatus de proporcionibus, donde describe el proceso de selección de ciencias demostrativas incluidas en el dominio de la aritmética ${ }^{22}$. En esa publicación se

21 S S. J. Livesey, "The Oxford Calculators, Quantification of Qualities and Aristotle's Prohibition of Metabasis", en: Vivarium, 1986 (24), p. 57.

22 S. J. Livesey, "The Oxford Calculators, Quantification of Qualities and Aristotle's Prohibition of Metabasis”, en: Vivarium, 1986 (24), p. 58. 
plantea la utilización de proporciones aritméticas en las que figuran cantidades de naturaleza diversa. Puesto que, en sentido estricto, una proporción matemática consiste en una igualdad de dos cocientes tal como $7 / 8=14 / 16$, en los que las cuatro cantidades son números sin otro significado aparte del estrictamente aritmético. Ahora bien, la noción de proporción así considerada planteaba la duda sobre la licitud de admitir como verdaderas proporciones aquellas en que las cantidades tenían un significado físico; por tanto, no estrictamente aritmético. Por ejemplo, las proporciones establecidas entre los valores que procedían de comparaciones o de medidas de magnitudes físicas, tales como la velocidad o la fuerza de resistencia al movimiento.

En un principio, Bradwardine consideró que en tales situaciones no era válido formular ese tipo de razones de proporcionalidad. Es decir, dentro de los principios establecidos por la aritmética, no era admisible igualar un cociente formado por dos valores numéricos (ambos de naturaleza estrictamente matemática) con otro cociente formado por dos cantidades cuyos significados no fueran matemáticos. Es evidente que en el fondo de este planteamiento latía la idea excluyente de ciencia aristotélica, la cual establecía una absoluta separación entre aritmética y cinemática, que eran consideradas materias pertenecientes a géneros diferentes. En definitiva, no era científicamente correcto combinar en la misma igualdad cantidades de diferente naturaleza. 
Posteriormente, Bradwardine rectificó su concepción inicial adoptando un enfoque menos estricto. Se alejó del terreno matemático y dio a la proporción un significado analógico. De este modo, la proporción entre cantidades servía para comparar, por ejemplo, las intensidades de dos sonidos.

Si no hubiera proporción entre propiedades porque no son cantidades (del mismo género) por la misma razón no habría proporción entre tonos y entonces, toda la ciencia de los armónicos se destruiría ${ }^{23}$.

En consecuencia, había que renunciar al significado exclusivamente numérico para utilizar la aritmética como instrumento de cálculo aplicado a la ciencia del sonido. Superada esta nueva dificultad derivada de la proscripción aristotélica sobre el papel que la ciencia debía desempeñar en el conocimiento de la naturaleza, la filosofía natural se aproximó un poco más al ideal de la ciencia experimental, afianzándose el razonamiento deductivo y el rigor lógico que imponía la matemática. En especial la geometría fue incorporada como ciencia auxiliar al estudio de la naturaleza, en parte, gracias a las observaciones sobre la transmisión de la luz realizadas por Robert Grosseteste.

23 S. J. Livesey, "The Oxford Calculators, Quantification of Qualities and Aristotle's Prohibition of Metabasis", en: Vivarium, 1986 (24), p. 59. 
Naturaleza y Libertad. Revista de estudios interdisciplinares. Número 4, 2014. ISSN: 2254-9668

\section{METAFÍSICA Y GEOMETRÍA DE LA LUZ}

Las investigaciones de Grosseteste realizadas en el siglo XIII son un ejemplo elocuente de la transición gradual que se produce desde las categorías filosóficas a los conceptos científicos cuantificables. Por un lado, aplicando nociones metafísicas, Grosseteste atribuía a la luz una naturaleza primordial o sustancia primera. Por otro lado, la luz dotada de propiedades geométricas, configura el espacio donde se hallan prefigurados todos los sucesos físicos. Se propaga por sí misma adoptando una forma geométrica y en virtud de su naturaleza se difunde en el espacio, se refleja en las superficies y se refracta al pasar de un medio a otro. Fenómenos naturales que pueden describirse utilizando conceptos y propiedades geométricas, tales como líneas rectas y ángulos que definen las trayectorias y las desviaciones sufridas por la luz al atravesar medios trasparentes, como una esfera de vidrio.

De aquí, estas reglas, principios y fundamentos han sido dados por la capacidad de la geometría. Por este método, la atenta observación de las cosas naturales puede proporcionar las causas de todos los efectos naturales. Y sería imposible de otro modo, como ya es claro en cuanto a lo universal, ya que cada acción natural expresa su variación en fortaleza y en debilidad a través de la variación de las líneas, de los ángulos y de las figuras. Pero, en lo que se refiere a lo particular, esto es incluso más claro, primero en la acción natural sobre la materia y después sobre los sentidos ${ }^{24}$.

24 A.C. Crombie, Robert Grosseteste and the origins of experimental science 1100-1700, Oxford at the Clarendon Press, 1962, p. 110. 
Según esta teoría, la descripción científica de los fenómenos ópticos es compatible con una visión filosófica de la naturaleza de la luz. Y, en virtud de propiedades luminosas, Grosseteste pretende dar razón de las dimensiones del espacio y de las "esferas del universo", atribuyendo a la luz el papel de causa eficiente de todo movimiento, mediante una combinación de nociones metafísicas y descripciones geométricas.

Además del efecto luminoso asociado a la luz, existe también el calorífico cuya medida Grosseteste trató de cuantificar estableciendo correlaciones entre las intensidades luminosas y las caloríficas, basándose en el ángulo de incidencia de la luz. Y en esta misma línea otros estudiosos de la óptica medieval, como Witelo analizaron la variación del ángulo de refracción de un haz luminoso con la variación del ángulo de incidencia (en un rango entre 10 y 80 grados) cuando atraviesa medios transparentes diferentes, como, aire, agua y vidrio, atribuyendo a las diversas densidades de los medios atravesados, las variaciones del ángulo de refracción ${ }^{25}$. Asimismo, el conocido fenómeno del arco iris proporcionó a los estudiosos medievales de la naturaleza una experiencia científica asequible. Pudieron realizar medidas de los ángulos que forman, con la visual del observador, las direcciones de los rayos solares al incidir en gotas de lluvia. Posteriormente, esa refracción natural fue 
reproducida artificialmente en el laboratorio, utilizando botellas de vidrio de forma esférica.

Otros autores medievales, como Teodorico de Freiburg reformularon las teorías aristotélicas sobre el color y la combinación de luz y oscuridad con el fin de obtener relaciones numéricas. Así, por vía experimental establecieron correlaciones entre el ángulo de refracción de un haz de luz y la posición que ocupaban los diferentes colores del espectro luminoso, desde el color rojo, en un extremo, hasta el azul, en el opuesto.

Estas breves referencias de esas primeras investigaciones permiten concluir que la óptica medieval trató de explicar mediante parámetros numéricos y relaciones geométricas las observaciones naturales.

Estos ejemplos ponen de manifiesto que para llegar a las nociones físicomatemáticas mensurables, es preciso realizar una reconstrucción experimental del fenómeno y someterlo a condiciones de control y medida para centrarse en las propiedades que se analizan. En la mayoría de los casos, los medios experimentales necesarios excedían las posibilidades de las universidades medievales. Los recursos técnicos más accesibles eran los destinados a las medidas y a la realización de cálculos numéricos que precisaban las observaciones astronómicas. También, los que se utilizaban en diversas aplicaciones, tales como en la elaboración de calendarios, en la navegación, o 
Naturaleza y Libertad. Revista de estudios interdisciplinares. Número 4, 2014. ISSN: 2254-9668

en los análisis y ensayos químicos de materiales con fines comerciales ${ }^{26}$. Pero fue precisamente mediante la experimentación cómo se consiguió someter al análisis matemático los aspectos cualitativos de la naturaleza.

\section{DE LA CUALIDAD A LA CANTIDAD}

Según la metafísica aristotélica, cantidad y cualidad son categorías irreductibles. O bien, no es posible expresar las variaciones cualitativas de una propiedad observable, como el color, en función de valores cuantitativos. En el siglo XII, había sido motivo de discusión la irreductibilidad de esas dos categorías aristotélicas aplicadas a la teología ${ }^{27}$.

\footnotetext{
En efecto, el origen de la controversia fue una crítica a la física cualitativa de Aristóteles a partir de dos puntos de vista derivados de fuentes griegas. Estos fueron primero, los conceptos físicos pitagóricos o platónicos que tomaban las diferencias cualitativas como reducibles a diferencias de naturaleza geométrica, numérica y de movimiento, esto es, reducibles a diferencias cuantitativas y secundariamente, a ciertos conceptos matemáticos y procedimientos $^{28}$.
}

26 A. C. Crombie, "Quantification in Medieval Physics”, en: Isis, 1961 (52, 2), p. 156-8.

$27 \mathrm{El}$ interés de los filósofos escolásticos por el problema de intesio y remissio de las cualidades tiene su origen en cuestiones teológicas debatidas por Pedro Lombardo en sus "Sentencias". S. T. Kirschner, "Oresme on Intension and Remission of Qualities in His Commentary on Aristotle's 'Physics", en: Vivarium, 2000 (38), p. 256.

28 A. C. Crombie, "Quantification in Medieval Physics”, en: Isis, 1961 (52, 2), p. 145. 
Así pues, la radical separación conceptual entre cualidad y cantidad impedía la asignación de valores numéricos a las variaciones cualitativas, restringiendo el horizonte de la ciencia experimental, al quedar fuera de la investigación aquellas propiedades que sólo son apreciables como sensaciones cualitativas, tales como, las sensaciones térmicas de calor y frío, o bien las producidas por impresiones luminosas o acústicas.

Afortunadamente, acabó imponiéndose el criterio experimental sobre el metafísico y se encontró el medio de expresar las variaciones cualitativas en función de cambios cuantitativos. Roger Bacon fue uno de los autores que contribuyó a este nuevo enfoque de la cuestión, pues renunciando a la teoría aristotélica, propuso la sustitución de las categorías filosóficas de naturaleza y forma por nociones geométricas.

Todas las categorías (praedicamenta) dependen del conocimiento de la cantidad, en relación con los rasgos matemáticos y por tanto toda la capacidad de la lógica depende de las matemáticas ${ }^{29}$ [Cursiva añadida].

De esta forma, la cantidad quedaba más estrechamente sujeta a las propiedades sensibles y se daba un paso más hacia la descripción cuantitativa de la naturaleza.

29 Opus Maius. (Ed. J. H. Bridges Oxford (1897), I. 103). Citado por A. C. Crombie, “Quantification in Medieval Physics”, en: Isis, 1961 (52, 2), p. 147. 
También la misma experiencia sensible proporciona un fundamento legítimo para expresar variaciones cualitativas en función de variaciones cuantitativas. Ya que, las sensaciones cualitativas que recibimos de los cuerpos están vinculadas a la cantidad, puesto que siempre sedan circunscritas a una porción de materia. Por ejemplo, el color está ligado a la superficie del cuerpo donde inhiere. Aunque la naturaleza del color no viene determinada por su extensión, sin embargo, está sujeto a ella y no sería percibido sin la existencia de esa superficie material. De igual modo, las propiedades asociadas a los fenómenos luminosos, tales como el brillo, la intensidad luminosa (todas ellas percibidas como cualidades) están supeditadas a propiedades geométricas.

Para hacer efectiva la medida experimental de variaciones cualitativas en función de valores cuantitativos, la ciencia medieval debía encontrar un método experimental apropiado, que permitiese asignar de modo indirecto valores numéricos a los cambios cualitativos, ya que de hecho la medida directa sólo es posible para las variaciones cuantitativas. Así, por ejemplo, la altura de un edificio es mensurable porque es divisible en partes de igual tamaño, todas ellas de la misma naturaleza. Es decir, los incrementos o decrementos de la cantidad son fracciones de la misma naturaleza que el conjunto. En ese caso, el resultado de la medida es un número (sea entero o fraccionario), que corresponde a las veces que la unidad de medida está contenida en el conjunto. 
Por el contrario, una cualidad, como el color, no puede descomponerse en porciones homogéneas. Su intensidad no aumenta o disminuye por adicción o sustracción de partes homogéneas; por el contrario, y a diferencia de la cantidad, varía gradualmente. Un color no es divisible en partes iguales; no es posible extraer de esa sensación visual una parte que sirva como unidad de medida. No sería imaginable, por ejemplo, una porción de color azul.

A su vez, las operaciones de medida de cantidades hace posible aplicar operaciones aritméticas que conducen a formulaciones matemáticas. Por el contrario, las variaciones cualitativas, tales como un aumento o disminución de calor, de sonido, de brillo luminoso, etc., no pueden ser registradas mediantes operaciones aritméticas, por lo que la intensidad resultante de dos sonidos, por ejemplo, no es la suma aritmética de cada una de las intensidades individuales. Análogamente cabe afirmar respecto al calor, a la iluminación, o al color.

Dentro de este intento por expresar las propiedades cualitativas en función de variaciones cuantitativas, algunos autores medievales idearon los "grados de intensidad" ${ }^{30}$ que aplicaron a las sensaciones térmicas. Y, de acuerdo con este modo de concebir las variaciones térmicas, el incremento de calor se explicaba como la acción combinada de un aumento de "grados de calor" y una disminución de "grados de frío".

30 S. T. Kirschner, "Oresme on Intension and Remission of Qualities in His Commentary on Aristotle's 'Physics”, en: Vivarium, 2000 (38), p. 259. 
A esta primera tentativa le siguieron otras que se vieron favorecidas por la mejora de técnicas y métodos experimentales. La experimentación permitió la realización de operaciones de medida de las variaciones cualitativas. Por ejemplo, basándose en la dilatación o contracción de la longitud (propiedad cuantitativa) de una delgada columna de mercurio provocada por las variaciones de calor (propiedad cualitativa), es posible construir escalas termométricas y, en definitiva, se obtiene una medida indirecta de efectos caloríficos.

Es evidente que las operaciones de medida experimental, al asociar valores numéricos, tanto a las propiedades cuantitativas, como a las cualitativas, abren el camino a la utilización de las matemáticas como ciencia instrumental, y con ello, la ciencia experimental es capaz de formular mediante expresiones matemáticas los fenómenos de la naturaleza ${ }^{31}$. En consecuencia, mediante el álgebra o el análisis infinitesimal, los procesos naturales se presentan bajo la forma de funciones numéricas, o bien leyes empíricas, que suelen llamarse "leyes de la naturaleza" (lex naturae), según la denominación 
Naturaleza y Libertad. Revista de estudios interdisciplinares. Número 4, 2014. ISSN: 2254-9668

de Roger Bacon ${ }^{32}$, con el mismo sentido que recibieron a partir del siglo XVII.

Las leyes de la reflexión y de la refracción son comunes a todas las acciones naturales, como lo he mostrado en el tratado de geometría ${ }^{33}$.

En la pretensión de describir la naturaleza mediante valores numéricos, tuvo una especial significación el fenómeno del movimiento que fue objeto de estudio por parte de algunos filósofos medievales vinculados a las Universidades de Oxford y de París.

\section{LA CUANTIFICACIÓN DE LA FÍSICA MEDIEVAL}

Entre los investigadores que abordaron el estudio del movimiento, se destacaron los Calculators del Merton College oxoniense ${ }^{34}$.Esta institución

32 Roger Bacon fue el primer autor que utilizó la expresión "ley de la naturaleza”. A. C. Crombie, "Quantification in Medieval Physics”, en: Isis, 1961 (52, 2), p. 147 - 8.

33 Un fragment inédit de l'OpusTertium (Ed. P. Duhem Quaracchi (1990); cf. P. 78). Citado en A. C. Crombie, "Quantification in Medieval Physics”, en: Isis, 1961 (52, 2), p. 148.

34 Existen amplias referencias a la historia de esta institución. Así, Hastings Rashdall, The Universities of Europe in the Middle Ages (Ed. F. M. Powicke and A. B. Emden, 3 vols. Oxford 1936, Vol 3, 191-201); Marshall Clagett, The Science of Mechanics in the Middle Ages, Madison, Wisc., 1959; A. G. Molland, "The Geometrical Background to the "Merton Sch001"', British Journal for the History of Science (1968), pp. 108-125. E. D. Sylla, The Oxford Calculators and the Mathematics of Motion 1320-1350. Physics and Measurement by Latitudes, 
Naturaleza y Libertad. Revista de estudios interdisciplinares. Número 4, 2014. ISSN: 2254-9668

universitaria fue fundada en 1264 por Walter Merton para albergar a estudiantes de la facultad de teología. Posteriormente, en el siglo XIV, surgieron algunos teólogos que sobresalieron en el cultivo de la filosofía natural y de las matemáticas. Entre ellos, Richard Swineshead que fue fellow entre 1344 y 1345 y escribió un Liber Calculationum o Calculationes, en el que expuso varios métodos de cálculo matemático ${ }^{35}$.

$\mathrm{Al}$ referirse a estas publicaciones medievales, conviene precisar que no deberían calificarse de estudios propiamente científicos en el sentido actual, sino más bien de pre-científicos. Pues, en algunos casos consistían en anotaciones y comentarios destinados a la enseñanza. En otros casos, como ocurre con Descriptiones motuum de Roger Swineshead y de la Summa de John Dobleton, ambos filósofos mertonianos, se trata de informes sobre problemas, como el del movimiento. Además, existían otros estudios destinados a la solución de cuestiones lógicas, tal como el titulado Regulae solvendi sophismata cuyo autor William Heytesbury escribe acerca de algunos métodos lógicos destinados a resolver dificultades gnoseológicas y al movimiento lo$\mathrm{cal}^{36}$.

New York, Garland, 1991. (Citados en S. J. Livesey, "The Oxford Calculators, Quantification of Qualities and Aristotle's Prohibition of Metabasis”, en: Vivarium, 1986 (24), p.50).

35 E. D. Sylla, The Oxford Calculators and the Mathematics of Motion 1320-1350. Physics and Measurement by Latitudes, New York, Garland, 1991, p. 19.

36 E. D. Sylla, The Oxford Calculators and the Mathematics of Motion 1320-1350. Physics and Measurement by Latitudes, New York, Garland, 1991, pp. 22-23. 
Dentro del campo de la filosofía natural, los temas abordados por los Calculators fueron muy variados ${ }^{37}$. Entre los cuales, se encuentran sencillos tratados matemáticos destinados a estudios introductorios de astronomía y de óptica; muchos de ellos se limitaban a recoger resultados orientados a la enseñanza. Además, suele observarse una descuidada presentación formal, junto con análisis incompletos de los temas. En buena parte, esas publicaciones provenían de notas procedentes de los debates académicos que, al ser objeto de apresuradas revisiones e interpolaciones, menoscababan su unidad interna. Finalmente, en cuanto a la forma expositiva, destaca la frecuente utilización de razones ad hominem y pruebas ad hoc, muy alejadas del tipo de argumentación científica posterior.

No obstante, la física medieval contribuyó notablemente a la descripción matemática del movimiento local. En dicha contribución tuvo mucho que ver la aparición de un nuevo contexto filosófico promovido por el pensamiento de Ockham que impulsó el desarrollo de la ciencia moderna ${ }^{38}$. En este nuevo ambiente filosófico el pensamiento científico gozó de mayor autonomía y, al prescindir de categorías aristotélicas, tales como géneros, especies,

37 E. D. Sylla, The Oxford Calculators and the Mathematics of Motion 1320-1350. Physics and Measurement by Latitudes, New York, Garland, 1991, pp. 26 - 34.

38 J. Sánchez Navarro, “Los experimentos imaginarios de Ockham a Galileo", en: Galileo y la gestación de la ciencia moderna. Acta IX. Encuentros, Canarias, 2001, pp. 63-80, p. 69. 
Naturaleza y Libertad. Revista de estudios interdisciplinares. Número 4, 2014. ISSN: 2254-9668

esencias, etc., centró el análisis científico sobre aspectos concretos de los fenómenos naturales, facilitando así el estudio experimental de la naturaleza.

En muchos casos los temas de filosofía natural procedían de cuestiones que se habían suscitado en un contexto teológico. En este caso, por exceder el ámbito propio de la ciencia sagrada, el autor debía elaborar su propia teoría fuera de ese campo, por lo que su pensamiento derivaba hacia la ciencia de la naturaleza. Así por ejemplo, Pedro Lombardo se planteó el cambio cualitativo al abordar el problema del aumento o disminución de la virtud de la caridad ${ }^{39}$.

Buena parte de la investigación en el campo de la filosofía natural que realizaron autores mertonianos, tales como Bradwardine, Heytesbury y Swineshead se centraba en el llamado "problema de la intensificación y disminución de formas y cualidades". Se admitía que la ganancia o pérdida de una especie o forma daba lugar a un aumento o disminución de una cualidad (como el color o el sabor), permaneciendo el mismo el sujeto después de la alteración cualitativa. Asimismo, la filosofía nominalista de Ockham aportó un marco conceptual que favoreció la medición de propiedades cualitativas, tales como las luminosas, mediante variaciones cuantitativas. Ya que, la intensidad luminosa (propiedad cualitativa) varía en función de diferentes ángulos de

39 A. C. Crombie, "Quantification in Medieval Physics”, en: Isis, 1961 (52,2), p. 151. 
inclinación de la luz (propiedad cuantitativa mensurable). Análogamente, el método era aplicable a las observaciones térmicas ${ }^{40} \mathrm{y}$ cromáticas.

Aparte de los fenómenos ópticos, también el movimiento local contribuyó al desarrollo del análisis cuantitativo de la naturaleza. Especialmente en el siglo XIV y XV, algunos filósofos de las universidades de Oxford y París se destacaron por sus estudios sobre el movimiento. En este campo, la aportación más notable consistió en establecer una correspondencia entre las diferentes posiciones que ocupa el cuerpo móvil y los sucesivos instantes del tiempo transcurrido. Los términos utilizados son todavía propios de la filosofía escolástica, pero con un significado diferente. Así, los "Calculadores" dieron el nombre de forma a cualquier cualidad o cantidad susceptible de cambio. La variación de dicha forma se medía por la intensio o latitud. Análogamente, la intensio del tiempo representaba la medida de la forma temporal. Estableciendo una correspondencia entre la intensio espacial y la intensio temporal, los movimientos se clasificaban en tres tipos: uniforme, disforme y uniformemente disforme $e^{41}$. Un móvil tiene un movimiento uniforme cuando recorre espacios iguales en tiempos iguales; lleva movimiento disforme cuando son diferentes los espacios recorridos y los lapsos de tiempo transcurridos; y,

40 Debido a la diferente inclinación de los rayos solares que llegan a la superficie de la Tierra, durante su movimiento alrededor del Sol, se produce una variación en el calentamiento de la atmósfera, en cada hemisferio terrestre.

41 A. C. Crombie, “Quantification in Medieval Physics”, en: Isis, 1961 (52,2), p. 153. 
Naturaleza y Libertad. Revista de estudios interdisciplinares. Número 4, 2014. ISSN: 2254-9668

el movimiento es uniformemente disforme (actualmente llamado uniformemente acelerado) cuando, en cada intervalo de tiempo, el espacio recorrido se incrementa la misma cantidad.

Ciertos autores, como Roger Swineshead desarrollaron una variante de este método empleando los conceptos de grados y de latitud de formas ${ }^{42}$ (en términos actuales, sería equivalente al rango de variación de un magnitud, como la velocidad). Por su parte, el mertoniano John Dumbleton asignó a la latitud de forma referida a la distancia, un significado cuasi geométrico, cercano a la noción moderna de la cinemática. Según esta última idea, latitud del espacio recorrido por el móvil implica la existencia de un medio continuo homogéneo y mensurable, como el espacio geométrico de la mecánica galileana. Pero el avance hacia la descripción matemática del movimiento fue aún mayor gracias a Nicolás de Oresme, filósofo de la Universidad de París, que desarrolló un método de representación de latitudes de formas ${ }^{43}$, utilizando esquemas gráficos, del tipo de las coordenadas en dos dimensiones. Según las cuales, en la dimensión vertical, la extensio venía representada por segmentos rectilíneos de longitud variable. Mediante este procedimiento gráfico, el au-

42 E. D. Sylla, The Oxford Calculators and the Mathematics of Motion 1320-1350. Physics and Measurement by Latitudes, New York, Garland, 1991, p. 65.

43 J. Sánchez Navarro, “Los experimentos imaginarios de Ockham a Galileo”, en: Galileo y la gestación de la ciencia moderna. Acta IX. Encuentros, Canarias (2001), pp. 63-80, p. 75. 
mento o disminución de la velocidad del móvil, se representa en función del tamaño de los segmentos verticales.

\section{TEORÍA MEDIEVAL DEL IMPETUS}

Como un caso particular del movimiento de un cuerpo, determinados autores vinculados a la Universidad de París trataron de explicar el movimiento parabólico recurriendo a la noción de impetus. Es decir, el movimiento descrito por un proyectil que, impulsado en el instante de salida, se desplaza sometido a la acción de la gravedad. Este tipo de movimiento tiene especial interés porque permite comparar el método utilizado por la ciencia medieval y el empleado posteriormente por Galileo.

El nombre de impetus fue acuñado por Jean Buridan, profesor de la universidad parisina. Con ese término se refería a la causa que actúa sobre el proyectil y le mantiene en el aire. En 1320 la teoría comenzó a tomar forma cuando Francisco de Marchia identificó esa causa con la que denominó vis derelicta, o fuerza capaz de mantener al proyectil en movimiento después de ser lanzado. Esta fuerza permanecía en el proyectil, por tanto, no estaba localizada en el aire como afirmaba Aristóteles y se iba debilitando paulatinamente hasta que desaparecía cuando el proyectil finalizaba su recorrido. Así pues, el término "permanente" aludía a una propiedad estable, ligada al proyectil durante el movimiento y, únicamente, era modificada o 
anulada totalmente, cuando actuaba otra fuerza externa, como la resistencia del aire o un obstáculo material interpuesto en la trayectoria del movimiento.

En consecuencia, según esta teoría medieval, el movimiento natural era debido al appetitus o inclinación radicada en la naturaleza misma de los cuerpos, mientras que, el movimiento violento de los proyectiles se explicaba en virtud del impetus ${ }^{44}$. Marshall Claggett se refiere a ese carácter permanente del ímpetu en los términos siguientes:

El impetus es una cosa permanente (res naturae permanentis), distinta del movimiento local en el que el proyectil es movido (...). Y es probable (verisimile) que el ímpetus sea una cualidad natural, es decir, físicamente presente y predispuesta para mover el cuerpo en el que está impresa, del mismo modo como se dice que existe una cualidad impresa en el hierro debido al imán que mueve al hierro hacia él. Y es también probable que de igual modo que esa cualidad, el ímpetu sea impreso junto con el movimiento por el motor en el cuerpo que se mueve y así también el ímpetu remite cuando el movimiento se destruye, o se impide por una resistencia, o por una inclinación contraria ${ }^{45}$.

Por tanto, según la ciencia medieval, el impetus es una cualidad propia de la naturaleza de los cuerpos materiales, en cierto modo, parecida a la propiedad magnética que poseen algunos minerales. Propiedad que reside en el

44 R. Fernández-Lomana del Río, "Dos principios para una axiología de la técnica”, en: Anuario Filosófico, 2007, p. 160.

45 S. Drake, "Impetus theory reappraised", en: Journal of the History of Ideas, 1975 (36,1), p. 32. 
proyectil de un modo latente y que se pone de manifiesto cuando es impulsado por un agente externo o motor que causa el movimiento. De acuerdo con estos mismos presupuestos Buridan pretende explicar el movimiento secular de los cuerpos celestes, cuyos desplazamientos no son impedidos por ninguna resistencia externa.

El ímpetu perduraría indefinidamente (in infinitum) si no disminuyera debido a una resistencia opuesta o por una inclinación a un movimiento contrario ${ }^{46}$.

Esta teoría medieval del ímpetu aplicada al movimiento de caída de graves, resulta ser especialmente significativa. En efecto, sobre el cuerpo que cae sometido a la gravedad actúan dos causas, por un lado el impetus y por otro su tendencia natural (pesantez) o atracción terrestre. La combinación de ambas causas da lugar a un movimiento uniformemente disforme (uniformemente acelerado). Conforme a la mentalidad medieval, esa descripción del movimiento, puramente matemática, no resultaba convincente y debía encontrase una explicación de carácter físico, que hiciese referencia a las dos causas que intervenían: la pesantez y el impetus. Evidentemente, tal explicación debía estar en consonancia con el esquema geométrico que representaba los incrementos discretos de velocidad en función de los intervalos de tiempo recorrido.

46 M. Clagett, The Science of Mechanics in the Middle Ages. Madison, 1951, p. 524. Citado por S. Drake, "Impetus theory reappraised", en: Journal of the History of Ideas, 1975 (36,1), p. 33. 
Según esa explicación, en el primer intervalo de tiempo sólo actuaba la gravedad, siendo el movimiento uniforme ${ }^{47}$ (con velocidad constante). A partir del segundo intervalo temporal actuaba también el ímpetu, el cual iba aumentando en los intervalos sucesivos, produciendo así un movimiento acelerado. La descripción del movimiento de caída se completaba haciendo intervenir el concepto de grados de velocidad ${ }^{48}$, que consistían en pequeñas variaciones discontinuas. De esta forma, en el primer instante del movimiento de caída, el móvil tenía 1 grado de velocidad, originado por la acción de la gravedad. En el segundo instante, tenía 2 grados de velocidad (uno debido a la gravedad y el otro al ímpetu). En el tercer instante, tenía 3 grados de velocidad (dos de ellos, debidos al ímpetu y el otro a la gravedad) y así sucesivamente. En definitiva, según la teoría medieval, el movimiento de caída libre se realizaba con una velocidad creciente, cuyo valor numérico resultaba ser proporcional al número ordinal asignado al intervalo de tiempo, comenzando a contar desde el origen del movimiento.

El ejemplo que acabamos de exponer es demostrativo de concepto de velocidad que tenían los autores medievales. Pues, revela que su noción del movimiento no era compatible con una variación continua de velocidad, por

47 Esa no es la interpretación correcta, pues la acción de la gravedad produce una aceleración constante (movimiento uniformemente acelerado) desde el mismo instante en que comienza el movimiento, como Galileo comprobó.

48 S. Drake, "Impetus theory reappraised", en: Journal of the History of Ideas, 1975 (36,1), p. 30. 
tanto, recurrían a incrementos finitos. Por el contrario, el concepto actual de variación instantánea de velocidad hace posible la utilización de funciones matemáticas continuas. Pero el concepto actual de "velocidad instantánea" supone admitir un cambio instantáneo en el valor de la velocidad, es decir, sin transcurso de tiempo. Esta noción, hoy día admitida por la ciencia moderna, era incompatible con la teoría medieval que pretendía explicar el movimiento acelerado basándose en incrementos discretos del ímpetu. Con carácter general, la física medieval no admitía la existencia de variaciones continuas de cualquier propiedad. Ni siquiera fue admitido por Galleo, según afirma Stillman Drake.

El concepto de una velocidad instantánea, completamente claro para nosotros, era una noción contradictoria para Galileo. La velocidad implica movimiento, y movimiento implica un lapso de tiempo, aunque sea pequeño. Aristóteles utilizó un término diferente, mutación, para el cambio que excluye el lapso de tiempo ${ }^{49}$.

La actual concepción del movimiento local, como cambio continuo de posición, equivale a admitir que la velocidad del móvil toma una infinidad de valores intermedios, a partir del instante inicial, lo que autoriza a equipararla con una función matemática dependiente del tiempo.

49 "Desde 1604 a 1608 Galileo pensaba en términos de velocidades referidas a muy pequeños intervalos de tiempo, no en velocidades matemáticamente instantáneas”. (S. Drake, Galileo: Pioneer Scientist, Toronto, University of Toronto Press, 1990, p. 103). 
Ante todo, es importante subrayar que desde el punto de vista metodológico, la idea de una variación instantánea no es algo que pueda extraerse directamente de la experiencia sensible, pues no es apreciable por los sentidos. Por el contrario, es fruto de una interpretación efectuada a partir de una representación idealizada del movimiento real, según la cual, la velocidad, el espacio recorrido, y el tiempo transcurrido se toman como entidades abstractas que varían como magnitudes matemáticas continuas. En consecuencia, las citadas propiedades físicas, velocidad, espacio y tiempo, se equiparan a las magnitudes matemáticas.

\section{LA NATURALEZA IDEALIZADA}

Las entidades físicas, como la velocidad, susceptibles de interpretación matemática son asimiladas a objetos matemáticos, consideradas como abstracciones o idealizaciones, construidas mediante la imaginación y, por tanto, no identificables con las meras percepciones sensoriales resultantes de la observación empírica.

Fueron algunos filósofos medievales de las Universidades de París y Oxford, los que aplicaron por primera vez ese recurso mental al razonamiento científico, al que calificaron como razonamiento secundum imaginationem.

Swineshead, entre otros autores del Merton College, utilizó el método secundum imaginationem con el fin de idear un sencillo experimento mental para utilizarlo como argumento en su teoría sobre la constitución del cosmos. El 
experimento mental consistía en imaginar una varilla muy fina que atravesando el centro de la Tierra podría alcanzar el centro del universo. Mediante esta imagen visual ideal, una vez libre de las restricciones impuestas por la materia, se pretendía impulsar el razonamiento discursivo, explorando posibles desarrollos y aplicaciones compatibles con la teoría. Para Steven J. Livesey, este experimento mental pretende ante todo comprender el alcance de la teoría:

Importa poco a Swineshead que este problema pueda ser inverificable o que la situación pueda ser realmente imposible. Es un problema enmarcado como secundum imaginationem, cuyo propósito es probar las condiciones de contorno de la teoría que se considera, no determinar cómo se comportan realmente las $\operatorname{cosas}^{50}$.

Es evidente que con este método el fenómeno tal como se observa pierde los detalles sensibles y se simplifica la complejidad asociada a los entes materiales individuales. Los rasgos abstraídos dejan de formar parte de un único individuo y pasan a ser atribuibles a cualquier otro objeto del mismo tipo. Así, el peso, como magnitud física abstracta, no es sólo una propiedad vinculada a un sólido concreto, pues se convierte en una propiedad compartida por todos los entes materiales sometidos a la atracción gravitatoria.

50 S. J. Livesey, “The Oxford Calculators, Quantification of Qualities and Aristotle's Prohibition of Metabasis", Vivarium (1986) 24, p. 64. 
Además, esos objetos idealizados, por ser inmateriales, no están sometidos a las restricciones materiales y pueden ser objeto de operaciones mentales, como lo son los objetos geométricos. Por ejemplo, un cuerpo sólido que ocupa una posición en el espacio podrá ser imaginado dotado de movimiento, por tanto, capaz de cambiar de posición con el transcurso del tiempo. Así mismo, se podrán establecer comparaciones con otros cuerpos idealizados, como se hace con las figuras geométricas.

La imaginación es el reino del transformismo o metamorfosis (...). Esta maleabilidad y docilidad de la imagen se debe a su menor vivacidad, a su tenuidad. Gana con lo que pierde; pero pierde con lo que gana. La imagen es dócil porque es asténica, espectral. La imagen, por su mayor tenuidad, es incuestionablemente menos precisa que la visión ocu$\operatorname{lar}^{51}$.

$\mathrm{Al}$ comienzo del siglo XIV la imaginatio estaba fuertemente asociada al pensamiento matemático ${ }^{52}$. La escolástica enseñaba que la matemática alcanza su "objeto material" mediante la imaginación, mientras que, la física lo

51 Así, literalmente, para "Aristóteles: la fantasía es una sensación asténica [asthenés]" (Retórica, 1.II, 1370 a 28) (J. Ortega y Gasset, La idea de principio en Leibniz. Madrid, Alianza Editorial, 1992, p. 100).

52 A. G. Molland, "The Geometrical Background to the 'Merton School”, British Journal for the History of Science, (1968) 4, pp. 113-114. Citado en S. J. Livesey, "The Oxford Calculators, Quantification of Qualities and Aristotle's Prohibition of Metabasis", Vivarium (1986) 24, p. 64, Nota 45. 
hace a partir de los sentidos, pues por su carácter empírico no puede prescindir de las impresiones sensoriales recibidas en la fase experimental. Estas impresiones serán posteriormente elaboradas para formar modelos o esquemas idealizados ${ }^{53}{ }^{54}$. De esta forma, los principios y las operaciones matemáticas pasan a formar parte de la ciencia experimental, aplicando su propia lógica interna a los datos extraídos mediante percepciones sensibles.

\section{LA CIENCIA EN GALILEO}

La ley de caída de graves obtenida por Galileo en 1604 es la primera verificación del método científico, en cuanto descripción matemática de los fenómenos naturales. Esa relación matemática establece cómo varía el espacio recorrido en función del tiempo, rechazando la pretensión de descubrir las causas internas que actúen en el movimiento. Se prescinde voluntariamente de toda explicación metafísica, partiendo únicamente de los datos

53 M. A. Herrero, Idealización en la ciencia y su papel en la construcción de las teorías científicas. Tesis Doctoral Facultad de Filosofía. Universidad Complutense de Madrid, 2010, p. 4.

54 Según M. W. Bundy, la imaginación media entre el sentido y la razón, primero, recreando las imágenes recibidas a través de los sentidos y después combinándolas para formar otras nuevas, lo cual permite considerar como un tipo de metabasis psicológica que sirve de transición entre dos diferentes reinos, el sensible y el racional. (The Theory of Imagination in Classical and Medieval Thought, Urbana, III, 1927. Citado en S. J. Livesey, "The Oxford Calculators, Quantification of Qualities and Aristotle's Prohibition of Metabasis", en: Vivarium, 1986 (24), p. 64, Nota 45. 
Naturaleza y Libertad. Revista de estudios interdisciplinares. Número 4, 2014. ISSN: 2254-9668

experimentales. Así lo manifiesta el científico italiano en el Diálogo, a través de uno de los personajes, haciendo referencia al movimiento acelerado.

Salviati. No me parece ocasión oportuna para entrar, al presente, en investigaciones sobre la causa de la aceleración del movimiento natural, en torno a la cual han sido diversas las opiniones emitidas por los filósofos, reduciéndola algunos a la atracción (avvicinamento) hacia el centro [de la Tierra], otros que van quedando sucesivamente menos partes del medio que ha de ser hendido, otros a cierta impulsión de parte del medio ambiente, el que al volver a reunirse por detrás del móvil, lo va oprimiendo y empujando continuamente. Sería interesante, aunque de poca utilidad, ir examinando y resolviendo todas estas fantasías y otras más. Por ahora a nuestro Autor le basta con que comprendamos que él quiere investigar y demostrar algunas propiedades de un movimiento acelerado (cualquiera que sea la causa de su aceleración), tal, que los aumentos de su velocidad vayan acrecentándose, después de su partida del reposo, en la misma simplicísima proporción en que crece la continuación del tiempo, que es lo mismo que decir que en tiempos iguales se lleven a cabo iguales aditamentos de velocidad; y si nos encontramos que con las propiedades que serán demostradas después se verifican en el movimiento de los graves naturalmente descendentes y acelerados, podremos juzgar que la definición adoptada comprende un tal movimiento de los graves, y que es verdad que su respectiva aceleración va creciendo según crece el tiempo y la duración del movimiento ${ }^{55}$ [Cursiva añadida].

Salviati manifiesta su pretensión de analizar los aumentos de su velocidad del movimiento acelerado, es decir, sus variaciones cuantitativas y excluye las

55 Galileo, Diálogos acerca de dos nuevas ciencias, Buenos Aires, Losada, 2003, p. 228; Galileo, Le Opere, Vol. 8, pp. 202-203. 
investigaciones sobre la causa de la aceleración del movimiento natural. Se comprende, por tanto, que Salviati no aluda a la teoría medieval del impetus, ya referida, pues eso implicaría admitir alguna causa intrínseca del movimiento. Así pues, el nuevo camino emprendido por la investigación científica se dirige a recoger los datos experimentales necesarios para establecer relaciones matemáticas entre los valores cuantitativos de magnitudes como la velocidad y el tiempo.

Eliminada la causa, como referencia metafísica, debemos tratar de comprender cómo consigue Galileo describir el movimiento, únicamente, mediante lenguaje matemático. Con ello, trataremos de aproximarnos a las claves de su método. Para lo cual, comenzamos señalando tres rasgos característicos que, a nuestro parecer, rigen el método galileano. En primer lugar y ateniéndonos a sus escritos científicos, destaca la utilización del procedimiento deductivo aplicado por Arquímedes a la resolución de problemas geométricos. En segundo lugar, subrayamos el uso frecuente de razonamientos secundum imaginationem que toman la forma de experimentos mentales ${ }^{56}$. Y, en tercer lugar, señalamos el modo de aplicar el método geométrico a problemas me-

56 La psicología renacentista en sus dos variantes, aristotélica y platónica, habrían servido de fundamento al uso de la imaginación que hizo Galileo para incorporar las matemáticas a la ciencia física. "Las matemáticas y la imaginación, que ocupan posiciones paralelas entre la sensación y el puro intelecto, fueron así equiparadas en ambas teorías [física y matemática]" (L. J. Daston, “Galilean analogies: Imagination at the bounds of sense”, en: Isis, 1984 (75), p. 305. 
cánicos. Los rasgos mencionados sintetizan los tres aspectos del método científico y proporcionan al estudio de los fenómenos físicos la capacidad deductiva de la geometría. Con el fin de mostrar con más detalle estas tres notas características, nos vamos a referir a un ejemplo estudiado en la mecánica galileana.

Le Mecaniche recoge un conjunto de estudios que corresponden a las lecciones sobre mecánica impartidas entre 1592 y 1610 por Galileo en Padua. Su estilo expositivo sigue el que utilizan en sus tratados de geometría Euclides y Arquímedes. Así al comienzo de la geometría euclídea, se definen nociones simples, tales como punto, recta, o ángulo, que servirán para construir figuras geométricas complejas. De forma análoga, la mecánica de Galileo establece definiciones y suposiciones a modo de principios fundamentales. "Como de semillas fecundas, brotan y nacen consecuentemente las causas y verdaderas demostraciones de las propiedades de todos los instrumentos mecánicos" ${ }^{57}$.

1) La gravedad de un cuerpo es la inclinación natural que tiende a moverse al centro de la tierra;

2) El momento es la inclinación del cuerpo cuando no sólo se considera el cuerpo sino también conjuntamente la situación que tiene en el brazo de la palanca;

57 M. Mersenne, Les Mechaniques de Galilée, Edición crítica de B. Rochot, Paris, Presses Universitaires de France, 1966, p. 26. 
Naturaleza y Libertad. Revista de estudios interdisciplinares. Número 4, 2014. ISSN: 2254-9668

3) El centro de gravedad es el punto respecto del cual todas las partes de él están en equilibrio o equiponderadas; de modo que si se imagina que el cuerpo se suspende de ese punto (centro de gravedad) las partes del cuerpo a la derecha se equilibran con las de la izquierda; las de atrás con las de delante, y las de arriba con las de abajo. Es decir, el cuerpo estará completamente en equilibrio y no se inclinará ni a un lado ni a otro ${ }^{58}$.

En consecuencia, mediante el método geométrico aplicado a la mecánica, Galileo deduce las propiedades que caracterizan las llamadas máquinas simples (palanca, polea, plano inclinado, etc.) y a partir de ellas explica los principios que rigen su funcionamiento.

La segunda nota del método galileano que hemos destacado, se refiere al papel que juega el razonamiento secundum imaginationem, según la expresión medieval. Es característico de su método y aparece indirectamente mencionado en el tercer párrafo de la referencia anterior, al definir el centro de gravedad de un cuerpo. Allí, Galileo invita al lector a formar una "representación idealizada" imaginando un cuerpo en equilibrio que está suspendido de un punto mediante un hilo. Por ejemplo, un sólido material de forma esférica concebido como una esfera geométrica, por tanto, susceptible de estudio geométrico, hace posible que la determinación del centro de gravedad se convierta en un problema estrictamente geométrico. $\mathrm{Y}$, en el caso de ser una

58 M. Mersenne, Les Mechaniques de Galilée, Edición crítica de B. Rochot, Paris, Presses Universitaires de France, 1966, pp. 26, 27. 
Naturaleza y Libertad. Revista de estudios interdisciplinares. Número 4, 2014. ISSN: 2254-9668

esfera homogénea, el centro de gravedad coincidirá con el centro geométrico de esa figura.

En definitiva, el método galileano utiliza la geometría como ciencia auxiliar para analizar los fenómenos mecánicos. Para ello, los cuerpos materiales que se presentan a la observación directa son idealizados, de forma que puedan ser equiparados a objetos geométricos. Al eliminar las limitaciones sensibles que impone la materia a los cuerpos reales, se consigue que la ciencia empírica participe del rigor deductivo que proporciona el razonamiento matemático. Con ello, el desarrollo de la mecánica fue posible debido a la construcción de modelos idealizados de tipo geométrico, susceptibles de ser analizados mediante principios y operaciones geométricas. La mente científica y filosófica de Galileo le llevó a comprender que el fundamento de la mecánica residía en la geometría.

Pero, dado que todas las leyes de la mecánica tienen sus fundamentos en la geometría, en la que no veo que el tamaño grande o pequeño de los círculos, triángulos, cilindros, conos o cualquier otra figura sólida afecte a sus propiedades, si la máquina más grande se fabrica de forma que todas sus partes están en la misma proporción que las de la pequeña siendo ésta fuerte y resistente para el trabajo a que se le destina- no veo por qué no ha de ser capaz de resistir los contratiempos adversos y destructivos que le puedan acaecer ${ }^{59}$ [Cursiva añadida].

59 Galileo, Le Opere, Vol. 8, p. 50. 
Puesto que la geometría se ocupa de las "relaciones cuantitativas" entre las dimensiones de los objetos y no de la "cantidad de materia", el funcionamiento mecánico depende de las relaciones de proporcionalidad entre las piezas que componen las máquinas.

El tercer rasgo característico ya señalado es de carácter operativo y se refiere al modo de aplicar los dos anteriores. Trataremos de analizar de qué forma el método galileano consigue integrar el razonamiento geométrico en la física del movimiento.

El mejor modo de ilustrar este aspecto será recurriendo a un pasaje recogido en la Jornada Tercera de los Discorsi, que comienza aludiendo a los movimientos locales. Después del movimiento uniforme, se aborda el análisis del movimiento acelerado. Galileo, fiel al método deductivo, enuncia veintidós teoremas con sus correspondientes demostraciones. En algunos casos seguidas de otros desarrollos, tales como escolios, corolarios y problemas. Está claro que no pretende simplemente adoptar el formalismo y la terminología propios de los tratados de geometría. Ante todo, trata de adaptar un modo expositivo a los principios mecánicos estructurándolos more geométrico para servir de fundamento al desarrollo deductivo posterior ${ }^{60}$. Ahora bien, como

60 Expresado en forma metafórica J. Daston escribe: "La aparente invisibilidad de las matemáticas per se en los escritos de Galileo puede deberse no sólo a su predominante interés físico, sino también al hecho de que las matemáticas están presentes bajo un ropaje físico" (L. 
se ha mencionado, esa estructuración exige previamente imaginar que un cuerpo material en movimiento puede reducirse a un punto geométrico, prescindiendo por tanto de sus determinaciones materiales. Análogamente, se toma el "concepto matemático de tiempo", como una magnitud de naturaleza geométrica que, a todos los efectos, juega el papel de una magnitud matemática que varía de forma continua.

Más concretamente, refiriéndose al movimiento acelerado, Galileo enuncia la Proposición 6, del Teorema VI, en los siguientes términos:

Si desde el punto más alto o más bajo de un círculo vertical sobre la horizontal, se trazan algunos planos inclinados hasta tocar la circunferencia, los tiempos de los descensos por los mismos serán iguales ${ }^{61}$.

Es decir, se plantea un estudio mecánico — un tanto artificial— desde un punto de vista matemático. Esto es, el movimiento de un cuerpo material que desliza por un plano inclinado liso. Y trata de demostrar que la velocidad final del cuerpo es la misma independientemente de los ángulos de inclinación de cada de unos los planos inscritos en una circunferencia, como la que

J. Daston, "Galilean analogies: Imagination at the bounds of sense”, en: Isis, 1984 (75), p. 305.

61 Galileo, Diálogos acerca de dos nuevas ciencias, Buenos Aires, Losada, 2003, p. 253; Galileo, Le Opere, Vol. 8, p. 221. 
se muestra en el esquema de la Figura 1 (adaptada del original de Le Opere de Antonio Favaro).

El texto del enunciado anterior combina elementos geométricos, como círculo y circunferencia, con términos mecánicos, como los planos inclinados y el tiempo. Esta combinación de elementos geométricos y físicos en plano de igualdad, es posible en virtud de la idealización de los entes físicos que son imaginados como entes geométricos. Así, un "plano inclinado", que en su realidad material, puede describirse como una superficie plana de madera, inclinada formando un cierto ángulo con la horizontal y que soporta un cierto cuerpo esférico, será representada idealmente por una simple porción de recta oblicua. En consecuencia, los planos pierden sus connotaciones materiales e incluso son reducidos a una sola dimensión siendo representados como las cuerdas $\mathrm{AB}$ y $\mathrm{AC}$ en la parte izquierda de la Figura 1, y las DA y $\mathrm{CA}$ en la parte derecha.

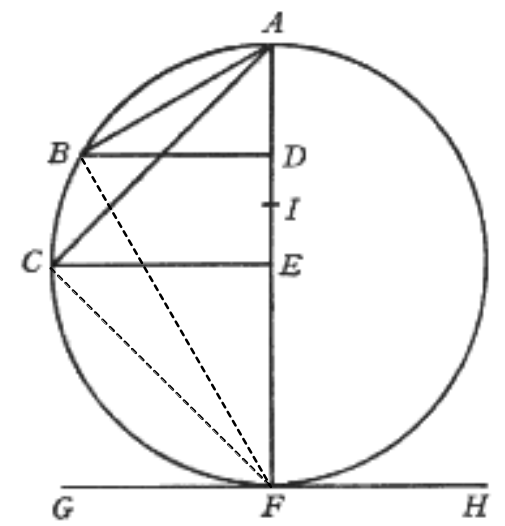

H

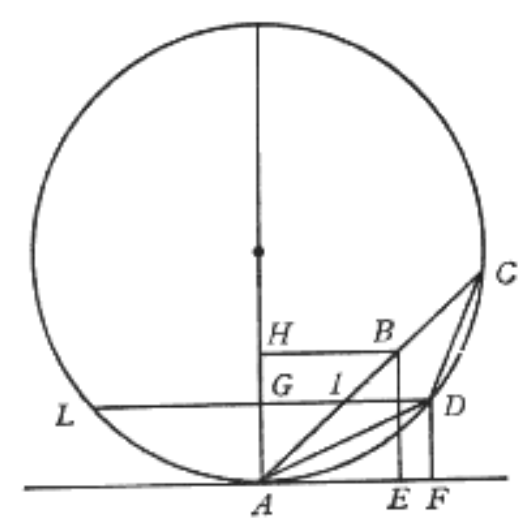

Figura 1 
Así pues, todos los elementos físicos que intervienen en el movimiento son expresados como objetos geométricos y en consecuencia, la demostración de la Proposición enunciada puede hacerse mediante operaciones matemáticas, tanto para el esquema de la izquierda que se refiere a los planos que parten del punto superior A de la circunferencia, como para el otro esquema de la derecha. Y de acuerdo con la interpretación física, el texto del enunciado afirma que un cuerpo tardaría el mismo tiempo en recorrer la distancia $\mathrm{AB}$, que en recorrer la longitud $\mathrm{AC}$. O bien, análogamente, en la figura de la derecha, el cuerpo tardaría lo mismo recorriendo CA que descendiendo por DA. La demostración seguida por Galileo se basa en el antiguo concepto matemático de media proporcional. Puesto que nuestro propósito es sólo ilustrar como se realiza la aplicación del método, parece más oportuno y más sencillo comprobar (no demostrar) la Proposición 6, utilizando la conocida ley de caída libre de cuerpos pesados, lo que se detalla en la Nota a pie de página $^{62}$. Para lo cual según el esquema de la izquierda de la Figura 1, comparamos la trayectoria inclinada $\mathrm{AB}$ y la $\mathrm{AC}$.

62 Tomando el descenso del cuerpo por el plano $A B$, será $A B=1 / 2 g(\operatorname{Cos} \alpha) t_{1}{ }^{2}[1]$, siendo g el valor constante de la aceleración de la gravedad y $\alpha$ es el ángulo formado por $\mathrm{AB}$ y $\mathrm{AFy} \mathrm{t}_{1} \mathrm{el}$ tiempo empleado en recorrer el diámetro DC. Análogamente, por el plano AC, la distancia $\mathrm{AC}=1 / 2 \mathrm{~g}(\operatorname{Cos} \beta) \mathrm{t}_{2}{ }^{2}$ [2], llamando $\beta$ el ángulo formado por CA y AF. Pero, en virtud de la relación geométrica que existe entre el diámetro de una circunferencia y cualquiera de sus cuerdas, resulta que $\mathrm{AB}$ es igual a $\mathrm{AF} \operatorname{Cos} \alpha$ y $\mathrm{AC}$ es igual a $\mathrm{AF} \cos \beta$. Por consiguiente, al sustituir en las igualdades respectivas [1] y [2], se tiene que el tiempo $t_{1}$ será igual a $t_{2}$. Es 
En definitiva, la aplicación del método al ejemplo mecánico estudiado, ha exigido, en una primera fase, la previa idealización geométrica de los elementos físicos que intervienen, mediante la eliminación de ciertas características materiales, tales como el rozamiento del plano inclinado $\mathrm{u}$ otros rasgos propios como el color o el brillo de la superficie. En la segunda fase, la estricta aplicación de los principios matemáticos ha conducido a la resolución del problema en términos geométricos. En una tercera fase, con la interpretación física del resultado ya obtenido, se tiene la conclusión expresada en términos mecánicos.

\section{LA CUANTIFICACIÓN DE LA CIENCIA EMPÍRICA. CONSIDERACIONES} FINALES

El método puesto en práctica por Galileo supuso un giro copernicano en la exploración científica de la naturaleza. Pues, Copérnico ideó un sistema solar que, además de superar al antiguo modelo de Ptolomeo, fue capaz de explicar los posteriores descubrimientos planetarios. Análogamente, el método galileano, no sólo resolvió los problemas mecánicos anteriormente

decir, el móvil tarda lo mismo en recorrer la longitud $\mathrm{AB}$ que la AC. Igualmente sucede con cualquier otro tramo inclinado que se considere comprendido entre $\mathrm{A}$ y cualquier punto de la circunferencia, pues todas las cuerdas de la circunferencia trazadas desde A cumplen la misma condición geométrica. Análogamente se demuestra para el esquema de la derecha de la Figura 1. 
Naturaleza y Libertad. Revista de estudios interdisciplinares. Número 4, 2014. ISSN: 2254-9668

planteados, sino que también abrió el camino que deberían seguir futuras investigaciones.

Para comprender cómo fue posible tal revolución en la ciencia experimental, debemos volver a Aristóteles y en concreto a su distinción entre episteme y techne $e^{63}$. La ciencia heredera de la episteme aristotélica descartaba la experimentación y analizaba los fenómenos naturales a partir de la mera observación, sin realizar manipulación técnica alguna que tuviera fines experimentales. Sin embargo, la ciencia empírica pudo progresar gracias al desarrollo de los instrumentos necesarios para realizar medidas experimentales.

Aun así, siendo la experimentación un factor necesario en el nacimiento de la "nueva ciencia", no fue por sí sola, una condición suficiente, como lo demuestran los resultados obtenidos por Galileo. Más bien, fue la asociación efectiva de la investigación experimental y de la geometría - y posteriormente, otras ramas de la matemática - lo que explica el fundamento del método galileano y su éxito en la descripción de los fenómenos naturales. En esa combinación de datos experimentales y de formalismo matemático, éste constituye el lenguaje simbólico necesario para "leer el gran libro de la naturaleza”, según la conocida metáfora galileana.

63 A. C. Crombie, “Quantification in Medieval Physics”, en: Isis, 1961 (52,2), p. 146. 
En el estudio de la mecánica, el lenguaje ideado por Galileo se basa primordialmente en el concepto de momento ${ }^{64}$, cuyo término procede a su vez del lenguaje común.

El momento, para los mecánicos, significa esa virtud, esa fuerza, esa eficacia con la que el motor mueve y el móvil resiste; tal virtud no depende sólo del peso sino de la velocidad y de las diversas inclinaciones de los espacios en los que se produce el movimiento, porque tiene más ímpetu un peso que desciende por un plano más inclinado que por otro de menor inclinación. Y en suma, cualquiera que sea la causa de tal virtud, recibe el nombre de momento. No me parece que este sentido pueda juzgarse nuevo en nuestra lengua ${ }^{65}$ puesto que, si no estoy equivocado, me parece que frecuentemente decimos: "Este es un negocio serio, pero este otro es de pequeño momento (importancia)", y "consideramos las cosas ligeras y pasamos a las que son de momento (importancia) ${ }^{66}$.

La definición de momento mecánico tiene su origen en la ley de la palanca de Arquímedes y combina en una misma magnitud, la longitud de uno de los brazos de la palanca y el peso aplicado en uno de sus extremos. Esta construcción ideal (resultado de una palanca real idealizada) se convierte en una herramienta conceptual, con la que Galileo estudia diversos artilugios mecánicos. Para ello, sigue el procedimiento señalado consistente en aplicar los

64 P. Galluzzi, Momenti: studi galileiani. Edizioni dell' Ateneo e Bizzarri. Roma (1979).

65 En una de las novelas ejemplares de Cervantes se lee: "No es este caso de tan poco momento" con el sentido de "no es cosa de tan poca importancia (momento)." M. Cervantes, Novelas Ejemplares, Barcelona, Círculo de Lectores, 2005, p. 55.

66 Galileo, Le Opere, Vol. 4, p. 68. 
principios de la geometría a los objetos mecánicos idealizados. Es evidente que este recurso mental implica realizar una transferencia de elementos procedentes de un contexto tecnológico al ámbito puramente conceptual ${ }^{67}$.Las aplicaciones del momento mecánico realizadas por Galileo, se extienden a la hidrodinámica y a la resistencia de materiales, mediante dos tipos de momento; uno estático y otro dinámico ${ }^{68}$.

Así pues, con esta breve referencia histórica sobre la definición de momento, ante todo, interesa subrayar la conexión entre la observación empírica (en este caso, mecánica) y la formulación teórica (geométrica), cuyo fruto del enlace entre teoría y observación empírica es el momento mecánico. Por lo cual, cabe afirmar que la formación de conceptos válidos para la descripción de fenómenos naturales no es el resultado de un proceso exclusivamente deductivo. Esto es, para llegar a una definición conceptual útil en la investigación científica, no es admisible la deducción lógica, tal como sucede con los teoremas matemáticos.

67 P. Yuste, "Reflexiones sobre la geometría griega", en: Endoxa, 2009 (23), p. 80.

68 Una palanca de brazos desiguales considerada en equilibrio estático sirve para establecer la proporción $\left(\mathrm{P}_{1} / \mathrm{P}_{2}=\mathrm{d}_{2} / \mathrm{d}_{1}\right)$ entre los pesos y las distancias al punto de apoyo o fulcro. Cuando los brazos de la palanca, inicialmente en equilibrio, oscilan ligeramente respecto al punto de apoyo, los pesos situados en los extremos describen un arco de circunferencia cuyo centro está en el fulcro y lo hace con velocidad mayor el que describe el arco mayor. De ahí se deriva una proporción entre velocidades y distancias $\left(\mathrm{v}_{1} / \mathrm{v}_{2}=\mathrm{d}_{1} / \mathrm{d}_{2}\right)$. 
En consecuencia, en virtud del vínculo que se establece entre experimentación y formulación teórica, las matemáticas aplicadas a los objetos materiales idealizados, se aproximan a los problemas reales, es decir, aquellos relativos a los cuerpos materiales, tales como el movimiento u otras manifestaciones mecánicas observables. En un principio, fue la geometría y posteriormente sería el álgebra, más tarde, el análisis infinitesimal, cuyo descubrimiento se atribuye indistintamente a Newton y a Leibniz.

El éxito del lenguaje matemático en la descripción de los fenómenos de la naturaleza se debe, ante todo, a la capacidad de realizar abstracciones sucesi$\operatorname{vas}^{69}$, con el fin de abordar los nuevos problemas planteados.

Los griegos sabían muy bien que una línea geométrica es la idealización de un cable físico a cuyo diámetro no nulo se le ha permitido idealmente reducirse a cero. Arquímedes tenía más o menos la idea de un punto material, en el sentido del límite "ideal" de una pequeña esfera de radio variable pero masa fija cuyo radio se ha reducido a cero. Los griegos se fabricaron la idea abstracta de un triángulo "general" a partir del conjunto de todos los triángulos individuales, y análogamente las ideas de un cuadrilátero, pentágono, etc., generales. Llegaron más lejos incluso y crearon la idea de un polígono general, cuyo número

69 A este respecto, es oportuno el comentario de J. Arana: "La doctrina de los grados de abstracción que aquí se enuncia no tiene nada que ver con la teoría clásica, porque no depende de la índole de los contenidos "seleccionados" por el proceso abstractivo, sino por la posibilidad de ejercer una ulterior abstracción sobre el producto de la anterior y precisamente en el mismo sentido que se ejerció la primera”. (J. Arana, “¿Es la naturaleza un libro escrito en caracteres matemáticos?”, en: Anuario Filosófico, 2000 (33), p. 64). 
Naturaleza y Libertad. Revista de estudios interdisciplinares. Número 4, 2014. ISSN: 2254-9668

de lados quedaba indeterminado y podía ser incluso variable. Esta última idea resultaba ser ya una abstracción de una abstracción, pero era una relativamente fácil ${ }^{70}$.

Los conceptos del análisis infinitesimal, tales como el de limite de una sucesión numérica o el de derivada han servido a la cinemática para definir la noción de velocidad instantánea y de aceleración instantánea, obteniendo de este modo una descripción más exacta y operativa del movimiento.

El concepto matemático de derivada es un concepto fundamental, uno de los conceptos más creativos y fecundos del análisis y del conocimiento humano en general. Sin él no existirían las ideas de velocidad ni de aceleración, ni de momento, ni la de densidad de masa o de carga eléctrica o cualquier otro tipo de densidad, ni la de gradiente de un potencial y, por tanto, ningún concepto de potencial en ninguna rama de la física; no habría ecuación de ondas, ni mecánica, ni física, ni tecnología. $\mathrm{Nada}^{71}$.

Es interesante señalar que, a pesar de que las categorías metafísicas fueron abandonadas por la ciencia moderna, éstas surgen de nuevo cuando se indaga en el fundamento de algunas nociones del análisis infinitesimal. Pues, el concepto aristotélico de "infinito potencial" subyace en la definición matemática

70 S. Bochner, El papel de la matemática en el desarrollo de la ciencia, Madrid, Alianza, 1991, pp. 59-60.

71 S. Bochner, El papel de la matemática en el desarrollo de la ciencia, Madrid, Alianza, 1991, p. 256. 
Naturaleza y Libertad. Revista de estudios interdisciplinares. Número 4, 2014. ISSN: 2254-9668

de limite, y a través de él, en las definiciones de derivada e integral. Y gracias a esos artificios matemáticos, la física describe las propiedades de cuerpos finitos y sus interacciones espacio-temporales, trascendiendo así las efímeras impresiones sensoriales de la observación. Y, en contraste con la formalización cartesiana de la naturaleza, la construcción galileana del lenguaje matemático logra describir los fenómenos naturales incluyendo las propiedades dinámicas. Con ello, las leyes físicas no quedan reducidas a meras relaciones matemáticas y la física no se diluye en la matemática. Por el contrario en la mathesis universalis cartesiana, la realidad material pierde sus propiedades sensibles y sólo permanece la sustancia extensa con sus tres dimensiones geométricas.

La naturaleza de la materia o del cuerpo, tomado en general, en modo alguno consiste en una cosa dura, o pesada, o con color, o de cualquier otro modo que afecte a nuestros sentidos, sino que la naturaleza del cuerpo solamente reside en ser una substancia extensa en longitud, anchura y profundidad ${ }^{72}$.

El método galileano describe las cualidades sensibles por sus efectos mensurables. Por ejemplo, mediante el termoscopio construido por Galileo hacia 1592 se pudo detectar variaciones de temperatura midiendo los cambios de la longitud de una columna de aire contenido en un tubo. Igualmente, las propiedades geométricas de una barra construida con un 72 R. Descartes, Los Principios de la Filosofía, Madrid, Alianza, 1995, p. 75. 
determinado material permiten estudiar su resistencia mecánica al ser sometida a una fuerza ${ }^{73}$. De esta forma, es posible afirmar que el método de la ciencia moderna capta el contenido de las sensaciones, conformándolas según el molde cuantitativo que le es propio. Aquello que los sentidos captan como duro, pesado o coloreado, la ciencia empírica lo traduce en propiedades de carácter físico-matemático.

Este modo de entender la tarea del método científico discrepa del expresado por Ernst Mach (1838-1916). Para el físico y filósofo austriaco las sensaciones constituyen el objeto de conocimiento de la física. En su Análisis de las sensaciones se pregunta acerca de "cómo un proceso químico una corriente eléctrica, etc. puede engendrar este efecto singular, a saber: el color verde. (...) El análisis psicológico nos enseña en este punto que tal asombro no está justificado, puesto que el físico siempre opera con sensaciones ${ }^{74}$. Es decir, que, según Mach, las sensaciones se convierten — sin ninguna alteración- en datos científicos, viniendo así a constituir el componente primordial de la ciencia empírica. Por lo cual, en la práctica, no existe diferencia entre las impresiones sensoriales en cuanto percibidas por el sujeto y en cuanto consideradas como entes físicos.

73 Galileo, Diálogos acerca de dos nuevas ciencias, Buenos Aires, Losada, 2003, pp. 157 y ss.; Galileo, Le Opere, Vol. 8, pp. 151 y ss.

74 E. Mach, Análisis de las sensaciones, Barcelona, Altafulla, 1987, p. 39 [Cursiva añadida]. 
Naturaleza y Libertad. Revista de estudios interdisciplinares. Número 4, 2014. ISSN: 2254-9668

Un color es un objeto físico en cuanto le pensamos como dependiente de la fuente luminosa (otros colores, calores, espacios, etc.). Si le consideramos como dependiente de la retina (...) es un objeto psicológico, una sensación. Lo diferente en ambos casos no es la materia sino la dirección de nuestras investigaciones ${ }^{75}$.

En consecuencia, según la función que para Mach tienen los órganos sensoriales, éstos se limitarían a registrar las sensaciones que provienen de los objetos del mundo exterior $y$, sin añadir ni quitar nada, sin posterior modificación, serían incorporados al pensamiento como datos científicos que posteriormente serán analizados. Si el fenómeno que se trata de estudiar es de tipo físico, químico, o biológico, esos datos recibirán un tratamiento acorde con el método y con los fines de esas ciencias. Si, por el contrario, se pretende hacer un estudio psicológico, entonces, esas mismas inmutaciones orgánicas externas tendrán el carácter de sensaciones.

Frente a estas consideraciones cabe señalar que los órganos de los sentidos nunca se comportan como meros receptores y transmisores de sensaciones. Según la conocida ley de Fechner, sus respuestas no son directamente proporcionales a los estímulos que reciben. Además no reúnen los requisitos necesarios para mantener la objetividad e independencia que son imprescindibles en la ciencia empírica, ya que sus respuestas son diferentes de unos

75 E. Mach, Análisis de las sensaciones, Barcelona, Altafulla, 1987, p. 16. 
individuos a otros, dependiendo incluso de las circunstancias personales y ambientales de cada sujeto.

Se ha de admitir que las sensaciones producidas en nosotros, cuyo origen está fuera de nosotros, son modificadas por el pensamiento y objetivadas por él para que sean aptas en el análisis científico, ya que, éste encuentra su rigor y precisión en la objetividad de la medida de las magnitudes científicas. Es un requisito indispensable impuesto por la ciencia que los datos que se manejen sean independientes del sujeto, del método de medida empleado y, asimismo, de los instrumentos utilizados.

Se comprende por esto, que las sensaciones tal como llegan a nuestra sensibilidad deban ser transformadas en objetos de pensamiento. Es decir, las imágenes sensibles ${ }^{76}$ son sometidas a un proceso de idealización que las convierte en objetos científicos. Entonces, aquello que el sentido no puede

$76 \mathrm{El}$ paso del plano físico al plano lógico que realiza el sujeto científico se asienta en la relación de semejanza como principio de inteligibilidad de la naturaleza. A él se refiere A. Marcos sosteniendo que "existe cierta distancia y también cierta conexión entre el plano físico y el lógico, y que este tipo de relación se da gracias a la semejanza. Ahora bien la semejanza misma no puede ser entendida de un modo estático y monolítico, no debe ser vista como algo simplemente dado. (...). Así pues, hemos seguido la semejanza como una relación tríadica, siguiendo la inspiración de Peirce, en la que la actividad del sujeto es imprescindible para la intelección" (...). "Las semejanzas están en la naturaleza como posibilidades objetivas basadas en la génesis y pasan a ser actuales gracias a la acción de un sujeto" (A. Marcos, La relación de semejanza como principio de inteligibilidad de la naturaleza, en: F. Rodríguez Valls (Ed.), La inteligencia en la naturaleza, Madrid, Biblioteca Nueva, 2012, pp. 91, 92). 
Naturaleza y Libertad. Revista de estudios interdisciplinares. Número 4, 2014. ISSN: 2254-9668

alcanzar, lo logra la razón. Así lo señala Galileo celebrando el predominio de la razón sobre la sensibilidad.

Mi admiración no encuentra límite por la forma en que la razón en Aristarco y en Copérnico ha conseguido vencer a la sensación (...). Me maravilla cómo Aristarco y Copérnico (...) han confiado tanto en lo que la razón les dictaba ${ }^{77}$.

Por sí mismas, las sensaciones no producen conocimiento científico. El papel de la ciencia experimental es convertir esas impresiones de los sentidos en enunciados y leyes cuantitativas valiéndose del carácter deductivo de las matemáticas. Para lo cual, a partir de las observaciones experimentales, el sujeto científico define conceptos útiles que remiten al mundo de los fenómenos naturales. En consecuencia, cabe afirmar que la ciencia empírica es el resultado de una actividad creativa de la razón que transforma los datos de los sentidos superando así las meras impresiones sensibles.

Miguel Ángel Herrero Universidad Politécnica de Madrid

ETSI Telecomunicación Ciudad Universitaria s/n. 28034 Madrid maherrer@fis.upm.es

77 Galileo, Le Opere, Vol. 7, pp. 355, 362. 